\title{
Thoracoscopic Esophagectomy in Prone Position
}

\author{
Guy Bernard Cadière' ${ }^{1}$, Giovanni Dapri ${ }^{1}$, Jacques Himpens ${ }^{1}$, and Amin Rajan ${ }^{2}$ \\ ${ }^{1}$ Department of Gastrointestinal Surgery, European School of Laparoscopic Surgery, Saint-Pierre University Hospital, \\ Brussels, Belgium; ${ }^{2}$ Department of Gastroenterology and Endoscopy, CHIREC, Brussels, Belgium
}

\begin{abstract}
Introduction. Esophagectomy can be performed by different minimal invasive techniques. We report a technique of Ivor Lewis esophagogastrectomy with manual anastomosis performed by thoracoscopy in prone position. Readers are encouraged to view the streaming video that accompanies this article.

Case report. A 51-year-old man was consulted for adenocarcinoma of the distal esophagus without lymph nodes invasion. Anesthesia was realized using a double-lumen endotracheal tube. The procedure started with the patient supine, and five abdominal trocars were placed. Celiac lymphadenectomy, wide Kocher maneuver, and pyloroplasty were performed. A wide gastric tube was advanced through the hiatus into the right chest. Subsequently the patient was placed in prone position and three trocars (two 5-mm, one $10-\mathrm{mm}$ ) were placed in the 5th, 7th, and 9th right intercostal space. The intrathoracic esophagus was
\end{abstract}

dissected, and mediastinal lymphadenectomy with en bloc resection of the left inferior mediastinal pleura was performed. After sectioning the azygos vein, the esophagus was transected by scissors $1 \mathrm{~cm}$ cranially. A completely thoracoscopic manual double-layer anastomosis was performed using running sutures with PDS 2/0 (externally) and Maxon 4/0 (internally). Finally the patient was replaced supine; the gastric tube was fixed to the hiatus, and the specimen was retrieved by suprapubic incision.

Results. Thoracoscopy lasted 157' (anastomosis 40'), laparoscopy 160', and second laparoscopy 20'. Blood loss was $170 \mathrm{cc}$. The patient was discharged on postoperative day 6.

Conclusions. Thoracoscopy in prone position allows the surgeon to perform a thoracoscopic esogastric anastomosis completely manually using only three trocars and without selective lung desufflation.
Electronic supplementary material The online version of this article (doi:10.1245/s10434-010-1330-7) contains supplementary material, which is available to authorized users.

(C) Society of Surgical Oncology 2010

First Received: 22 November 2009;

Published Online: 23 October 2010

G. B. Cadière

e-mail: coelio@resulb.ulb.ac.be 